\title{
Hyperlipoproteinemia Type IIb
}

National Cancer Institute

\section{Source}

National Cancer Institute. Hyperlipoproteinemia Type IIb. NCI Thesaurus. Code C35637.

A disorder of lipoprotein metabolism characterized by high levels of cholesterol and triglycerides in the blood. It is caused by elevation of low density and very low density lipoproteins. 